Britain joining without making a retrospective contribution."

Cesarsky says that the ESO will enter negotiations with a flexible outlook. An offer in kind - for example, giving astronomers from ESO member states access to British telescopes at Paranal would be considered seriously. But to suggest that the ESO would reduce the total value of the entry fee is, she says, "pure dreaming".

Even if the question of the entry fee is settled, there is also the issue of the annual payments, which would currently amount to $£ 12$ million a year. Without a significant increase in government funding to cover this sum, there would have to be cuts in other parts of the PPARC's budget.

Some of the money could be found, for example, by closing some of Britain's existing telescopes on La Palma in the Canary Islands. This prospect is worrying astronomers working on the facilities. There is also concern from particle physicists that covering a commitment to the ESO could be achieved by taking money away from accelerator research.

But the importance to British astronomers of joining the ESO was underlined in a report, Unveiling the Universe, published by the PPARC last week. It lists joining the $\mathrm{ESO}$ as high among its priorities. Reflecting the priorities of the PPARC's astronomy panel, the report suggests that the research council should seek an extra $£ 39$ million from the government over two years.

Halliday is not only confident of success in his negotiations with the ESO, but also optimistic that his efforts to build closer European collaboration will be received sympathetically in Whitehall. "ESO is currently not a well funded organization," he says. "They probably have more telescopes than they can afford. Somewhere in there is a potential for negotiation."

However, Cesarsky says that the ESO is not so strapped for cash that it needs to compromise to suit Britain's pocket.

"Britain knows what the conditions are for joining," she says.

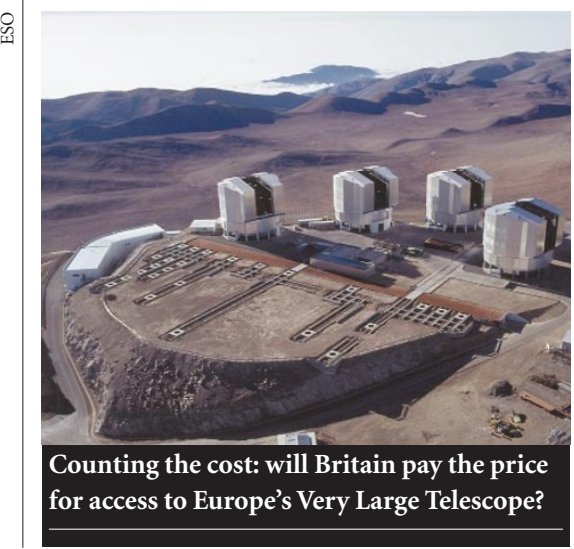

\title{
Mathematicians chase the seven million-dollar proofs
}

\section{David Dickson}

Mathematics might be the queen of the sciences, but as far as international prizes go, its omission from the list of Nobel disciplines has made it a poor relation.

All that, however, is about to change. The recently established Clay Mathematics Institute (CMI) in Cambridge, Massachusetts, was due to announce this week that it is offering \$1 million for the proof of each of seven classical mathematical problems.

Among them is the Riemann hypothesis concerning the distribution of prime numbers - widely considered the most important problem in number theory - and the Navier-Stokes equations familiar to anyone who has studied fluid dynamics.

Also listed is the ' $\mathrm{P}$ vs NP' (polynomial versus non-deterministic polynomial) problem. This concerns the relationship between the difficulty of solving a problem and of checking solutions, and bears directly on the theoretical possibility of being able to break computer codes (see Nature 400, 115-116; 1999).

"We wanted to make a statement to the general public that maths is important," says Arthur Jaffé, professor of mathematical physics at Harvard University and a member of the CMI's Science Advisory Board. "We believe that the future of the world is going to be dependent on science, and that mathematics is at the centre."

Jaffé was largely responsible for persuading businessman Landon T. Clay, 18 months ago, to set up a non-profit foundation dedicated to increasing and disseminating mathematical knowledge. The CMI already funds a range of activities, from research fellowships to workshops and summer schools. The institute now hopes that its decision to offer a series of prizes will recreate the type of public fervour that accompanied the solution of Fermat's last theorem in 1993 by the British-born mathematician Andrew Wiles.

"We wanted to do something original," says Alain Connes, professor of analysis and geometry at the Collège de France in Paris, which hosted the meeting at which the prizes were to be announced. Like Wiles, Connes is a member of the CMI's four-member Science Advisory Board.

It is almost a century since German mathematician David Hilbert set out 23 key unresolved problems that he predicted would set the agenda for mathematics in the new century, during a famous lecture in Paris, on 8 August 1900. His prediction was accurate. The problems he set out dominated much of mathematics in the past 100 years, and all but three have been resolved. (The solution of

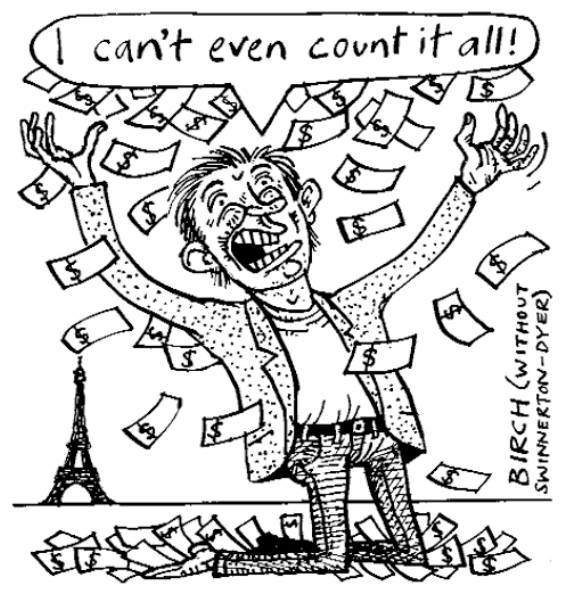

the Riemann hypothesis is one of the three outstanding problems from Hilbert's list.)

In contrast to Hilbert's speech - and reflecting concern in some quarters that the CMI might be attempting a similar task those responsible for the prize emphasize that they have no desire to determine the direction in which mathematics moves forward.

Rather, they are trying to recreate excitement about the activity of mathematics, both in the general public and among school students. "Sometimes people have the wrong idea of maths and think that it will be overtaken by computers," says Connes. "The seven problems, each selected by top specialists in their respective fields, are totally inaccessible to computers."

The competition has strict rules for determining whether a problem has actually been solved. In particular, a proposed solution must be published in a refereed journal "of worldwide repute", and must have gained "general acceptance" in the mathematics community within two years of publication. Also, to ensure that the lure of a reward does not encourage mathematicians to lock themselves away and work on the problems in private, prior work will be taken into account in awarding a prize.

There is no time limit to the competition. "We know that they are difficult, because people have been working on them for a long time," says Jaffé. But he also expresses optimism: "I would hope to live to see each of these solved."

The $\$ 1$ million problems are:

- The P vs NP problem

- The Riemann hypothesis

- The Poincaré conjecture

- The Hodge conjecture

- The Birch and Swinnerton-Dyer conjecture

- Navier-Stokes equations

- Yang-Mills theory

http://www.claymath.org 